\title{
Bündnis und Vertrag: zwei Grundmetaphern für die Ethik therapeutischer Berufe
}

\author{
Jede Beziehung zwischen Therapeuten (Ärzten, Pflegenden) und Patienten \\ hat moralische Grundlagen, die im Verlaufe der historischen Entwicklung der \\ Heilberufe verschiedene Formulierungen erfahren haben. Von besonderer \\ Bedeutung sind die Metaphern des Bündnisses und des Vertrags, die religiöse, \\ säkulare bzw. juristische Vorstellungen aufnehmen und sie auf die Therapeut- \\ Patienten-Beziehung übertragen. Während sich das Bündnisparadigma eignet, \\ in Situationen der Nicht-Reziprozität (Demenz, Urteilsunfähigkeit, Unmün- \\ digkeit) moralische Orientierungshilfen zu geben, eignet sich das Vertrags- \\ paradigma für reziproke Beziehungen, in denen ärztliches und pflegerisches \\ Handeln aufgrund eines «informed consent» ermöglicht ist.
}

\section{Settimio Monteverde*}

lic. theol. VDM, MAE, Anästhesiepfleger; Ethikkommission des Schweizerischen Berufsverbandes der Pflegefachfrauen und -männer; Kantonale Ethikkommission beider Basel

a Personenbezeichnungen gelten sinngemäss für beide Geschlechter.

Korrespondenz:

Settimio Monteverde, lic. theol.

VDM, MAE

Bethesda-Spital

Klinik für Anästhesie

Gellertstrasse 144

CH-4020 Basel

settimio.monteverde@bethesda.ch

\section{Therapeutisches Handeln als phronesis}

Die berufliche Pflege und Betreuung kranker Menschen stellt ein kulturhistorisch wichtiges Merkmal westlicher Zivilisationen dar. Als Motor der Etablierung von Heilberufen und eines differenzierten Gesundheitswesens fungierte zunächst eine primär religiös motivierte Tugendethik. Diese wurde sukzessiv durch die Entdeckung und Erforschung der biologischen Grundlagen des Menschseins und die ätiologische Einordnung von Pathologien erweitert. Das Bemühen um eine rationale Grundlage der Medizin, das für unseren Kulturraum spätestens im hippokratischen Schrifttum dokumentiert ist, hat eine bis in unsere Tage anhaltende Polarität zwischen der Medizin als Wissenschaft und der Medizin als Heilkunst heraufbeschworen, die beinahe anekdotisch immer wieder Gegenstand von Festreden an medizinischen Hochschulen ist. «Good medicine is a rational practice based on a scientific education and years of clinical experience. It is neither an art nor a science. Or, if it must be one of them, it must also be the other.» $[1,2]$. In Anlehnung an die Nikomachische Ethik von Aristoteles [3] beschreibt die US-amerikanische Medizinethikerin Kathryn Montgomery, dass es Medizin nicht mit episteme zu tun hat, also mit Erkenntnis von Dingen, die gleich Naturgesetzen unwandelbar sind [1]. Ebenso wenig ist sie techne, d.h. rein praktisches Können, das sich nur im Bereich des Wandelbaren bewegt. Sie ist hingegen phronesis, Klugheit, eine zur inneren Haltung gewordene Fähigkeit, die im Handeln Wertvolles und Werthaftes zu realisieren vermag. Phronesis ermöglicht es, situativ das Richtige zu erkennen und danach zu handeln. Folglich geht es nicht alleine darum, dass der Arzt ${ }^{\mathrm{a}}$ oder die Pflegefachperson das Richtige, dem «state of the art» Gemässe, tun, sondern um die Art und Weise, wie sie es tun.

\section{Religiöse und säkulare Begründungen therapeutischen Handelns}

Eine religiöse Begründung therapeutischen Handelns stellt das Beziehungsgeschehen zwischen Therapeut und Patient in einen grösseren Begründungszusammenhang, der solches - mitunter invasives - Handeln moralisch legitimiert. Die Regula Benedicti aus dem 6. Jahrhundert, die als geistliche Regel und programmatische Charta der Organisationsentwicklung des Benediktinerordens gilt, sieht im Dienst am Kranken einen besonderen Dienst an Christus. Dieser unterliegt sowohl für den eigens dafür beauftragten Mönch (infirmarius), aber auch für den Kranken (infirmus) selbst, besonderen Sorgfalts- und Verhaltensregeln. Während der Ordensgründer Benedikt von Nursia (480-547) ein grosser Förderer der Medizin war und die medizinischen Schriften des Altertums übersetzen liess, verbot Bernhard von Clairvaux (1090-1153), Gründer des Zisterzienserordens, die Beschäftigung seiner Mönche mit Medizin. Die ambivalente Haltung der Kirche gegenüber der Medizin, die sich - je nach Sichtweise - in einem Spektrum von der Nachfolge Christi bis zur Hybris bewegen konnte, setzt sich auch nach der methodologischen und epistemologischen Trennung der Medizin von der Theologie fort [4]. 
Als Anwendungswissenschaft ohne einen transzendenten Wertebezug konnte sich die Medizin nie mit der Erweiterung ihres Wissensbestandes allein begnügen. Schon die hippokratische Prognostik und Psychosomatik mussten zwar den Ansprüchen der Wissenschaftlichkeit genügen [5], sie waren aber nie Selbstzweck: Das Worum-willen, die teleologische Ausrichtung dieses Projekts, war von Anfang an mitgedacht [6]. Die ganzheitliche Betrachtungsweise einzelner Befunde, aber auch des Patienten in seinem biopsycho-sozialen Bezug, konstituieren seit Hippokrates die moralischen Grundlagen einer nunmehr säkularisierten Medizin: «La médecine n'est pas une démiurgie: sa finalité se limite à tout mettre en œuvre, en respectant la vulnérabilité de chaque malade, pour permettre à celui-ci de rétablir son équilibre compromis dans l'environnement qui est le sien.» [7]

Menschliches Handeln ist besonders begründungspflichtig, wenn es ein Handeln an anderen ist. Das Beschneiden individueller Freiheitsräume wird durch die Absicht des Therapeuten legitimiert, keinen Schaden zuzufügen, Gutes zu tun, um das Wohl des Patienten bekümmert zu sein. So verkündet es die paradigmatische Formulierung des hippokratischen Eides. Diese klassischen Grundlagen medizinethischer Reflexion wurden nach 1945, in der Aufarbeitung des krassen beruflichen Fehlverhaltens von Ärzten und Pflegenden unter dem Nationalsozialismus, erneut ins Bewusstsein gerufen. Die Nürnberger Rechtsprechung konnte zwar (noch) nicht auf kodifiziertes Recht zurückgreifen, doch die Richter gingen davon aus, dass fundamentale Prinzipien wie dasjenige des Nichtschadens und der Fürsorge einen quasinaturgesetzlichen Charakter hätten, der selbstverständlicher Bestandteil des Ärzteethos sei [8]. Dieses wurde im Nürnberger Codex, aber auch in der Erklärung des Weltärztebundes von Helsinki, um das Prinzip der Autonomie und der informierten Zustimmung (informed consent) erweitert.

In seinem ersten Buch «Bioethics - A Bridge to the Future» [9] geht der US-amerikanische Biochemiker Van Rensselaer Potter (1911-2001) angesichts ökologischer, atomarer und technologischer Bedrohungsszenarien der Frage nach, wie das wissenschaftliche Paradigma der Medizin um die Werte, die therapeutisches Handeln prägen, ergänzt werden könne. Er prägt für diese Art der Reflexion den Begriff der Bioethik (bioethics): Diese sieht er als Brücke, die den Wertebezug der Medizin herzustellen vermag [10]. Damit gelangt die von Aristoteles inspirierte Vision der Medizin, die primär phronesis, d.h. situationsgerechte Klugheit ist, zu einer Renaissance, wenngleich unter völlig neuen Umständen.

\section{Bündnis und Vertrag}

Phronesis als primäre Wertbezogenheit therapeutischen Handelns sagt freilich noch nicht aus, welches die Werte sind, die therapeutisches Handeln effektiv prägen. Diese Frage hat im Verlauf der Geschichte unterschiedliche Antworten erfahren. Bei Aristoteles sind es Tugenden (vgl. Fussnote c), d.h. Charakterdispositionen, die über die Performance einer Handlung etwas aussagen; ähnliches gilt für die christlich motivierte Pflege Kranker. Bei Hippokrates hingegen ist es eine ganzheitliche Vision des Gegenübers. In Anlehnung an theologische und philosophische Traditionen haben besonders zwei Konzepte eine grosse Bedeutung erlangt: der im alten Testament verankerte religiöse Bündnisgedanke und die in der Neuzeit entwickelte, säkulare Vertragstheorie der Moral. Die Situation des Bündnisses bzw. der Einigung und Unterzeichnung eines Vertrags bilden Metaphern, mit denen ein Wertebezug für ärztliches und pflegerisches Handeln hergestellt wird. In beiden wird der kranke Mensch, aber auch der Proband im Rahmen von Forschungsprojekten, als Kooperationspartner gesehen, mit oder für den etwas getan wird. Als Handeln zugunsten anderer [11] haben sowohl das Bündnis als auch der Vertrag den Charakter einer moralischen Konvention [12]. Der Inhalt der Vereinbarung hat normativen Charakter, d.h., die Handlung der Kooperationspartner ist in dem Masse gut, als sie mit den Inhalten des Bündnisses bzw. des Vertrags übereinstimmt. ${ }^{\text {b }}$

Das alttestamentliche Bündnis gründet im Heilshandeln Gottes zugunsten seines Volkes, in der Befreiung von der Knechtschaft und in der Konstituierung einer religiösen und politischen Gemeinschaft [14]. Der Bündnisgedanke operationalisiert sich sozusagen tugendethisch als Bündnistreue in Entscheidungen des klinischen Alltags [15]. ${ }^{c}$ Die säkulare Vertragstheorie der Moral stellt hingegen eine rationale Übereinkunft dar, in der Menschen aus freien Stücken die Art ihrer Beziehung verbindlich festlegen, die für beide Seiten von Vorteil ist [19]. Dieser Vorteil ist bei der Bündnisbeziehung nicht gegeben [20]. Somit ist Altruismus bzw. Eigeninteresse als Begründung moralischen Handelns ein wichtiges Unterscheidungsmerkmal zwischen Bündnis und Vertrag.

\section{Reziprozität der Beziehung}

Den Metaphern des Bündnisses und des Vertrags liegen zwei grundsätzlich verschiedene Konstellationen zugrunde, in denen sich therapeutisches Handeln ereignet:

1. Die auf den Bündnisgedanken zurückgreifende Konstellation ist durch Nicht-Reziprozität charakterisiert: Das Gegenüber des Therapeuten ist der Patient, der auf das Wissen und Können des Therapeuten angewiesen ist und in seiner 
durch die Krankheit bedingten Verletzlichkeit professionelle Hilfe aufsucht. Verletzlichkeit besagt hier, dass Patienten nicht fähig sind, ihre eigenen Interessen geltend zu machen, sei es, weil sie urteilsunfähig bzw. unmündig sind oder weil ihre Wünsche nicht frei von externer Beeinflussung sind [21]. Therapeutisches Handeln beruht in diesem Falle nicht auf Wechselseitigkeit und bewegt sich deshalb in einem moralisch sensiblen Bereich [22]. Solches Handeln erfährt eine «vertikale» Legitimation durch die übergeordnete Metapher des Bündnisses Gottes mit der Menschheit, des Bündnisses der Starken mit den Schwachen in der Gesellschaft, der Solidargemeinschaft von Gesunden und Kranken usw. Die Akteure (Ärzte, Pflegende, Patienten) handeln hier in einem Raum, der durch religiöse Prägungen, gesellschaftliche Werte und Erwartungen an das Gesundheitswesen geprägt ist. Diese haben sich für Pflegeberufe in den letzten Jahren kaum geändert - trotz radikaler Transformationen durch Professionalisierung, marktwirtschaftlicher Orientierung und Akademisierung [23].

2. Die Vorstellung des Vertrags hingegen geht von einer grundsätzlichen Reziprozität der beiden Partner aus: Das Gegenüber des Therapeuten ist hier der informierte Klient, der eine qualifizierte Dienstleistung in Anspruch nimmt und über die er sich mit dem Therapeuten im- oder explizit einigt. Moderne Theorien der Krankenpflege etwa greifen immer wieder den Vertragsgedanken auf. So z.B. diejenige von Dorothea Orem, die am Selbstfürsorgedefizit ansetzt und pflegerisches Handeln als erneute Ermöglichung von Selbstfürsorge definiert [24]. Hier greift therapeutisches Handeln subsidiär ein und ermöglicht eine klar beschreibbare, imoder explizit vereinbarte Leistung. Ist die Beziehung zwischen Therapeut und Patient wechselseitig, so bedarf es der juristisch hergeleiteten Metapher des Vertrags, um Rechte und Pflichten beider Kooperationspartner zu berücksichtigen. Jedes Mal, wenn Patienten und Probanden ihre informierte Zustimmung (informed consent) zur Beteiligung an einem Forschungsprojekt geben oder schriftlich/mündlich ihre Einwilligung für eine Intervention (operative oder medikamentöse Therapie, pflegerische Massnahme) erfolgt, bewegen sich die Akteure innerhalb des Vertragsparadigmas. Extremen des Paternalismus und der Indifferenz bildet das von Sally Gadow entwickelte Konzept der «advocacy», das sich der ebenfalls juristischen - Metapher des Fürsprechers bedient, der dem Klienten die eigenen Wertvorstellungen vergegenwärtigt und ihm dazu verhilft, diese in der Situation der Krankheit $\mathrm{zu}$ verwirklichen [28]. gespielt werden, denn sie haben grundsätzlich verschiedene Situationen vor Augen, die sich darin unterscheiden, ob die Beziehung des Therapeuten zum Patienten/Klienten reziprok ist oder nicht. Ist keine Reziprozität gegeben, d.h., besteht keine Möglichkeit, mit dem Patienten die Gestaltung der Therapie zu besprechen, so bedarf es für die moralische Rechtfertigung therapeutischen Handelns einer übergeordneten Idee, die gleichsam einen Raum eröffnet, in dem Handeln zu Gunsten des Gegenübers trotz mangelnder Einwilligungsfähigkeit möglich wird (Bündnis, Fürsorge, Achtsamkeit, «caring relationship» [25]).

Bündnis und Vertrag als komplementäre Paradigmen bergen aber auch Probleme: Das Machtgefälle zwischen Therapeut und Patient, das nicht-reziproke Bündnisbeziehungen oft charakterisiert [26], kann zu moralisch heiklen Situationen führen. Die normative Vorstellung eines Bündnisses konstituiert eine Gemeinschaft, in der der einzelne oft nicht gefragt wird, ob er deren Mitglied sein möchte. Sind es religiöse oder politische Vorstellungen, die ein solches Bündnis prägen, kann dies für den einzelnen, der sich in einen Kontext gesellschaftlicher oder religiöser Erwartungen hineingestellt sieht, die andere Menschen und Generationen für ihn geprägt haben, auch mit Konflikten assoziiert sein [27]. Zudem hat die Gender-Perspektive ein mitunter kritisches Licht auf historische Auswirkungen der Bündnistradition hinsichtlich der Stellung der Frau in Heilberufen geworfen. Ein Bündnis konstituiert immer eine Solidargemeinschaft. Diese droht dann zu einem huis clos zu werden, wenn sie nur unter Ausschluss weiterer konstitutiver Aspekte einer Situation zustande kommt (z. B. Kostenträger, Institutionen, politische Instanzen, evtl. weitere mögliche Begünstigte im Rahmen von Fragen der Verteilgerechtigkeit).

Das Bündnisdenken birgt einerseits die Gefahr des Paternalismus, der sich - im Rahmen einer das Bündnis begründenden Vorstellung über den aktuellen Wertekontext des Patienten hinwegsetzen kann. Andererseits stösst das Vertragsparadigma dort an seine Grenzen, wo der Klient in der Entscheidungsfindung letztlich alleine gelassen wird und - unter dem Vorwand der Eigenverantwortung - dieser in der Wahrnehmung derselben nicht unterstützt wird. ${ }^{\mathrm{d}}$

Eine qualitative Studie aus England, die die Verweigerung von Pflege von seiten der Patienten untersucht, kommt zu folgendem Schluss: «There is evidence in this study that nurses do not allow a patient to refuse nursing care. Participants in this study expressed a strong preference for securing the patient's agreement prior to carrying out care. They did not feel, however, that care should be omitted if the patient's agreement could not be 
achieved and the intervention was clinically indicated. In their view, consent can be described as desirable but ultimately not essential.» [29]. Eine Erklärung dieser Umstände könnte sein, dass ethische Konfliktsituationen dann entstehen, wenn der Therapeut (in diesem Fall die Pflegenden) die Beziehung zum Patienten von einem anderen Paradigma aus gestaltet als der Patient/Klient. Es bedarf deshalb einer Kontextsensitivität, die die Frage nach der Reziprozität der therapeutischen Beziehung situativ erfasst, Veränderungen wahrnimmt und diese konsequent in Entscheide miteinfliessen lässt [30]. Gerade wenn Patienten eingeschränkt urteilsfähig sind, ist es möglich, dass sie hinsichtlich bestimmter Fragen klar Auskunft geben können (z.B. bezüglich der Ernährung) und deshalb das Vertragsparadigma punktuell zur Geltung kommen kann, auch wenn dies für komplexere Zusammenhänge nicht mehr möglich ist. Somit besteht phronesis im Kontext therapeutischen Handelns darin, aufgrund der Frage nach der Reziprozität der Beziehung die Bündnis- bzw. Vertragsmetapher herbeizuziehen, um ärztliche und pflegerische Entscheidungen im klinischen Kontext zu begründen.

\section{Literatur}

1 Montgomery K. Phronesis and the Misdescription of Medicine: Against The Medical School Commencement Speech. In: Kuczewski MG, Polanski R (eds.). Bioethics - Ancient Themes in Contemporary Issues. Cambridge, MA: MIT Press; 2000. p. 57-66.

2 Thomasma D. Aristotle, Phronesis and Postmodern Bioethics. In: Kuczewski MG, Polanski R (eds.). Bioethics - Ancient Themes in Contemporary Issues. Cambridge, MA: MIT Press; 2000. p. 67-91.

3 Aristoteles. Nikomachische Ethik. Übersetzung von Franz Dirlmeier. Stuttgart: Philipp Reclam; 1986.

4 Breck J. Facing Bioethical Dilemmas Theologically. In: Messer N (ed.). Theological Issues in Bioethics. An Introduction with Readings. London: DartonLongman-Todd; 2002. p. 19-25.

5 Bergolt K. Das Gewissen der Medizin. Ärztliche Moral von der Antike bis heute. München: Beck; 2004.

6 Honnefelder L. Die ethische Entscheidung im ärztlichen Handeln. Einführung in die Grundlagen der medizinischen Ethik. In: Honnefelder L, Rager G (Hrsg.). Ärztliches Urteilen und Handeln. Zur Grundlegung einer medizinischen Ethik. Frankfurt a. M.: Insel; 1994. S. 135-90.

7 Célis R. Actualité de la pensée hippocratique. De la nécessité de fonder l'éthique médicale sur une épistémologie de la pratique soignante. In: Haldemann F, Poltier H, Romagnoli S (éds.). La bioéthique au carrefour des disciplines. Hommage à Alberto Bondolfi à l'occasion de son $60^{\mathrm{e}}$ anniversaire. Bern: Peter Lang; 2006. p. 149-76.

8 Jonsen AR. The Birth of Bioethics. New York; Oxford University Press: 1998.

9 Potter VR. Bioethics: Bridge to the Future. New York; Prentice Hall: 1971.

10 Whitehouse PJ. The Rebirth of Bioethics: Extending the original formulations of Van Rensselaer Potter. Am J Bioethics 2003;3(4):W26-W31.
11 Stemmer P. Handeln zugunsten anderer. Eine moralphilosophische Untersuchung. Berlin: de Gruyter; 2000.

12 Käppeli S. Das therapeutische Bündnis in Medizin und Pflege - wie lange noch? Schweiz Ärztezeitung 2006;87(26):1221-5.

13 Emanuel EJ, Emanuel LL. Vier Modelle der ArztPatient-Beziehung. In: Wiesing U (Hrsg.). Ethik in der Medizin. Ein Reader. Stuttgart: Philipp Reclam; 2000. S. 85-8.

14 May WF. The Medical Covenant: an Ethics of Obligation or Virtue? In: Messer N (ed.). Theological Issues in Bioethics. An Introduction with Readings. London: Darton-Longman-Todd; 2002. p. 156-71.

15 Ramsey P. Preface to The Patient as Person. In: Lammers SE, Verhey A (eds.). On Moral Medicine. Theological Perspectives in Medical Ethics. $2^{\text {nd }}$ edition. Cambridge: Nm. B. Eerdmans; 1998. p. 53-7.

16 Zimmermann-Acklin M. Tugendethische Ansätze in der Bioethik. In: Düwell M, Steigleder K (Hrsg.). Bioethik. Eine Einführung. Frankfurt a. M.: Suhrkamp; 2003. S. 200-10.

17 McKay AC. Supererogation and the profession of medicine. Symposium: Heroes - or just doing their job? J Med Ethics 2002;28:70-3.

18 Downie RS. Supererogation and altruism: a comment. Symposium: Heroes - or just doing their job? J Med Ethics 2002;28:75-6.

19 Pauer-Studer H. Einführung in die Ethik. Wien UTB; 2003.

20 Müller W-E. Argumentationsmodelle der Ethik. Positionen philosophischer, katholischer und evangelischer Ethik. Stuttgart: Kohlhammer; 2003.

$21 \mathrm{Jubb}$ AM. Palliative care research: trading ethics for an evidence. J Med Ethics 2002;28:342-6.

22 Schweizerische Akademie der Medizinischen Wissenschaften. Recht der Patientinnen und Patienten auf Selbstbestimmung. Medizinisch-ethische Grundsätze der SAMW. Basel: SAMW; 2005.

23 Heffels WM. Pflegeethik als Verpflichtung zur Wahrnehmung personaler Verantwortung der Pflegenden in funktionalisierten Handlungsfeldern der Pflege. Dissertation. Fakultät für Gesellschaftswissenschaften, Universität Duisburg; 2002.

24 Orem DE. Eine Theorie der Pflegepraxis. In: Schaeffer D, Moers M, Steppe H, Meleis A (Hrsg.). Pflegetheorien. Beispiele aus den USA. Bern: Hans Huber; 1997. S. 85-97.

25 Käppeli S. Bündnis oder Vertrag? Eine Reflexion über zwei Paradigmen der helfenden Beziehung. Pflege 2005;18:187-95.

26 Conradi E. Vom Besonderen zum Allgemeinen Zuwendung in der Pflege als Ausgangspunkt einer Ethik. In: Wiesemann C, Erichsen N, Behrendt H, Biller-Adorno N, Frewer A (Hrsg.). Pflege und Ethik. Leitfaden für Wissenschaft und Praxis. Stuttgart: Kohlhammer; 2003. S. 30-46.

27 Sperl D. Ethik der Pflege. Verantwortetes Denken und Handeln in der Pflegepraxis. Stuttgart: Kohlhammer; 2002.

28 Gadow S. Existential Advocacy: Philosophical Foundation of Nursing. In: Spiker Stuart F, Gadow S (eds.). Nursing - Images and Ideals. New York: Springer; 1990. p. 79-101.

29 Aveyard H. The patient who refuses nursing care. J Med Ethics 2004;30:346-50.

30 Schweizerischer Berufsverband der Pflegefachfrauen und -männer SBK. Ethik und Pflegepraxis. Bern: SBK; 2003. 